\title{
Two-dimensional Fermi liquid dynamics with density and quadrupolar interactions
}

\author{
Rui Aquino ${ }^{1}$ and Daniel G. Barci ${ }^{1}$ \\ ${ }^{1}$ Departamento de Física Teórica, Universidade do Estado do Rio de Janeiro, \\ Rua São Francisco Xavier 524, 20550-013 Rio de Janeiro, Brazil
}

(Dated: August 29, 2019)

\begin{abstract}
We consider a Fermi liquid model with density-density as well as quadrupolar forward scattering interactions parametrized by the Landau parameters $F_{0}$ and $F_{2}$. Using bosonization and a decimation technique, we compute collective modes and spectral functions for a huge range of interactions, ranging from strong repulsion to strong attraction in either angular momentum channels. We present a dynamical phase diagram showing a region of parameters where the collective modes structure changes abruptly, possibly signaling a dynamical phase transition.
\end{abstract}

\section{INTRODUCTION}

Quadrupolar interactions in Fermi liquids are increasingly been considered due to the possible relevance in understanding the strange metal behavior observed in several highly cor-


trigger an electronic isotropic-nematic quantum phase transition $^{2}$. This transition could be at the bottom of anisotropic transport properties observed in several materials, running from cuprates superconductors ${ }^{3}$, pnictides ${ }^{4}$, quantum Hall devices ${ }^{5}$ and heavy fermions systems ${ }^{6}$.

Even though a systematic classification of the isotropicnematic quantum critical point is still lacking, the order parameter dynamics is reasonably well understood. In particular, the most relevant contribution to the Fermionic dynamics at criticality seems to come from an overdamped collective excitation with cubic dispersion relation ${ }^{7}$. Moreover, superconducting instabilities in the presence of quadrupolar interactions open the possibility of very interesting states of mat$\operatorname{ter}^{8-10}$ with novel topological excitations combining fractional vortex and disclinations.

In the usual theory of Fermi liquids ${ }^{11}$, two-body forward scattering interactions are parametrized by Landau parameters $F_{\ell}$, with $\ell=0,1,2, \ldots$, where the index $\ell$ labels angular momentum representations. For instance, while $F_{0}$ parametrizes the isotropic density-density interaction, $F_{2}$ measures the intensity of quadrupolar interactions. In Ref. 7, a simple model with pure quadrupolar coupling was studied near the isotropic-nematic phase transition driven by the Pomeranchuk instability, $F_{2} \sim-1$. Interestingly, it was observed that the Goldstone mode (in the nematic phase) has a precursor in the disordered isotropic phase which dominates the dynamics near the phase transition.

With this motivation in mind, we present in this paper a detailed analysis of the collective modes structure of a more general model, taking into account density as well as quadrupolar interactions. We explore collective modes in a huge range of parameters, running from strong repulsive to strong attractive interactions in both angular momentum channels, up to the Pomeranchuk instability region.

By means of a two-dimensional Bosonization technique $^{12-19}$, we parametrize Fermi surface deformations in terms of a set of chiral Bosons. We write an evolution equation in the semiclassical approximation, analog to the Landau
Fermi liquid formalism. Using an angular momentum basis, the model is reduced to a set of infinitely coupled harmonic oscillators. Each oscillator describes a Fermi surface deformation mode with a specific symmetry. In this way, the model is mapped into a classical chain of harmonic oscillators, in which the angular momentum label, $\ell$, plays the role of lattice sites in a one-dimensional chain. Taking advantage of this structure, we are able to exactly compute Green functions using a recursive decimation procedure ${ }^{20}$; a particular implementation of the real space renormalization group theory ${ }^{21-23}$. By looking for Green functions singularities, we compute collective modes and spectral functions. In this way, we are able to build a dynamical phase diagram (Fig. 2) in the $\left(F_{0}, F_{2}\right)$ plane. We show that, in specific regions of this plane, the Fermi surface dynamics changes abruptly, signaling a dynamical phase transition.

The main results of the paper are displayed in Figs. 4 and 5 in which we show longitudinal as well as transverse quadrupolar spectral functions for different regions of the dynamical phase diagram (Fig. 2). In Fig. 4(a) we show the equivalent of the Landau zero sound for the quadrupolar interaction, expected to occur for repulsive couplings, while in the second panel, Fig. 4(b), an unexpected result is displayed. Due to the quadrupolar attraction, besides the Landau zero sound, another well-defined collective mode appears at high frequencies, clearly separated from the particle-hole continuum. As the attraction becomes stronger, these two energy levels approximate each other and finally melt in a single damped mode at a critical value of the Landau parameters, in which the Green function has a second-order pole. This is an example of a so called "exceptional point" in open quantum systems 24,25 . At these points, eigenfunctions properties, such as orthogonality and phase rigidity suddenly change and have been associated with a dynamical phase transition (DPT) ${ }^{26}$. This sudden change in the dynamics occurs in an extended region of the parameter space (white region in Fig. 2). For stronger attractive interactions, near the Pomeranchuk instability, the overdamped mode, which is present in the whole attractive region, acquires a huge spectral weight, as shown in Fig. 4(c). This mode is identified as the precursor of the nematic Goldstone mode, computed in Ref. 7. Finally, Fig. 5 displays the spectral function of transverse modes. This type of modes cannot exist in a Fermi liquid with isotropic interactions. However, they are possible when higher angular momentum components of 
the interactions are considered. Very recently, an equivalent excitation called share sound was computed in a Fermi liquid model with dipolar interactions $\left(F_{1}\right)^{27}$. For attractive interactions, the share mode is damped, acquiring an important spectral weight near zero frequency, in the Pomeranchuk instability region. In the rest of the paper we give details of the model and calculations that conduce to these results.

The paper is organized as follows: in Sec. [II, we briefly review the Bosonization approach to Fermi liquids while in Sec. III we explicitly present the model. In Sec. IV we compute the Green functions and in Sec. $\nabla$ we analyze the structure of the collective modes. In Sec. VI] we show the spectral functions in different regimes and we finally discuss our results in Sec. VII We have left to the Appendix $A$ a detailed description of the decimation technique to compute Green functions.

\section{FERMI SURFACE DYNAMICS}

In this section, we briefly review the bosonization approach to Fermi liquids, in order to establish notation, the model and the main approximations we use in the paper.

The Fermi surface deformation can be parametrized in terms of a set of $N$ chiral Bosons $\varphi_{S}(\mathbf{x}, t), S=1, \ldots, N$, where the index $S$ labels a patch of width $\Lambda$ and height $\lambda$ in which the Fermi surface was coarse-grained. All through the paper, we use bold characters to represent vector quantities. The location of the Fermi momentum is then written as

$$
\mathbf{k}_{S}(\mathbf{x}, t)=\mathbf{k}_{S}^{0}+\nabla \varphi_{S}(\mathbf{x}, t)
$$

where $\mathbf{k}_{S}^{0}$ is the original uniform Fermi momentum at the patch $S$. This parametrization should be understood in a semiclassical scheme, since it mixes momentum and configuration space in a coarse-grained scale $|\mathbf{x}| \gg 1 /\left|\mathbf{k}_{F}\right|$. To recover the original Fermi surface, it is necessary to perform the continuum limit $\Lambda \rightarrow 0, N \rightarrow \infty$ with $\Lambda N=2 \pi k_{F}$.

A generating functional can be constructed in a coherent state path integral formalism as ${ }^{14.17}$

$$
Z=\int \mathcal{D} \varphi e^{-i S\left[\varphi_{S}\right]}
$$

where the integration measure is $\mathcal{D} \varphi=\prod_{S} \mathcal{D} \varphi_{S}$. The action can be split into two terms, $S=S_{0}+S_{\text {int }}$. The first term codifies the free Fermionic dynamics and is given by

$S_{0}=\frac{N(0)}{2} \sum_{S} \int d^{2} x d t\left[-\partial_{t} \varphi_{S} \mathbf{v}_{S} \cdot \nabla \varphi_{S}-\left(\mathbf{v}_{S} \cdot \nabla \varphi_{S}\right)^{2}\right]$,

where $\mathbf{v}_{S}$ is the Fermi velocity in each patch $S$, pointing perpendicular to the surface. $N(0)$ is the density of states at the Fermi surface. The second one represents two-body forward scattering interactions and is given by

$$
\begin{aligned}
S_{\mathrm{int}}=\frac{N(0)}{2} \sum_{S, T} \int d^{2} x d^{2} x^{\prime} d t F_{S, T}\left(\mathbf{x}-\mathbf{x}^{\prime}\right) \times \\
\left(\mathbf{v}_{S} \cdot \nabla \varphi_{S}(\mathbf{x})\right)\left(\mathbf{v}_{T} \cdot \nabla \varphi_{T}\left(\mathbf{x}^{\prime}\right)\right) .
\end{aligned}
$$

$F_{S, T}\left(\mathbf{x}-\mathbf{x}^{\prime}\right)$ represents particle-hole pair interactions between patches $S$ and $T$. Since this is a quadratic theory, in principle, it has exact solution. Note, however, that the quadratic character has been obtained by linearizing the Fermionic dispersion relation around the Fermi surface. Curvature terms give rise to non-quadratic Bosonic interactions 19 . Even so these terms are important to stabilize Pomeranchuk instabilities, they are irrelevant in the Fermi liquid phase ${ }^{\underline{7}}$.

A semiclassical description is obtained by computing the Euler-Lagrange equations $\delta S / \delta \varphi_{S}=0$, with $S=1, \ldots, N$. They can be cast in terms of normal fluctuations

$$
\delta n_{S}(\mathbf{x}, t)=N(0)\left(\mathbf{v}_{S} \cdot \nabla \varphi_{S}(\mathbf{x}, t)\right),
$$

obtaining

$$
\frac{\partial \delta n_{S}(\mathbf{q}, t)}{\partial t}+\left(\mathbf{v}_{S} \cdot \mathbf{q}\right) \sum_{T}\left\{\delta_{S, T}+F_{S, T}(\mathbf{q})\right\} \delta n_{T}(\mathbf{q}, t)=0 .
$$

The diagonal term in the patch basis (the $\delta$-function in the second term) comes from the free Fermion dynamics, while interactions mixes patches all around the Fermi surface.

For simplicity, we will focus on a circular Fermi surface. In this case, and in the absence of external magnetic fields, the angular momentum basis is much more convenient than the patch basis. Using the fact that $\delta n_{S}$ and $F_{S, T}$ are periodic functions of $S$, we can write,

$$
\begin{aligned}
\delta n_{S}(\mathbf{q}) & =\sum_{\ell=-\infty}^{\infty} m_{\ell}(\mathbf{q}) e^{i \ell \theta_{S}} \\
F_{S, T}(\mathbf{q}) & =\sum_{\ell=-\infty}^{\infty} F_{\ell}(\mathbf{q}) e^{i \ell\left(\theta_{S}-\theta_{T}\right)}
\end{aligned}
$$

where $\cos \left(\theta_{S}\right)=\hat{n}_{S} \cdot \mathbf{q}$ and $\cos \left(\theta_{T}\right)=\hat{n}_{T} \cdot \mathbf{q}$. Thus, $\theta_{S}$ $\left(\theta_{T}\right)$ is the angle subtended by the particle-hole momentum $\mathbf{q}$ with the normal direction to the patch $\hat{n}_{S}\left(\hat{n}_{T}\right)$. Since $F_{S, T} \equiv$ $F\left(\left|\theta_{S}-\theta_{T}\right|\right)$, then $F_{\ell}=F_{-\ell}$. In terms of $m_{\ell}(\mathbf{q})$, the equation of motion reads,

$$
\frac{\partial m_{\ell}(\mathbf{q}, t)}{\partial t}+i \frac{v_{F} q}{2}\left[\alpha_{\ell-1} m_{\ell-1}(\mathbf{q}, t)+\alpha_{\ell+1} m_{\ell+1}(\mathbf{q}, t)\right]=0
$$

in which $\alpha_{\ell}(\mathbf{q}) \equiv 1+F_{\ell}(\mathbf{q})$.

It is convenient to define symmetric and antisymmetric variables,

$$
m_{\ell}^{ \pm}(\mathbf{q}, t)=\frac{1}{2}\left[m_{\ell}(\mathbf{q}, t) \pm m_{-\ell}(\mathbf{q}, t)\right]
$$

in such a way that $m_{-\ell}^{+}=m_{\ell}^{+}$and $m_{-\ell}^{-}=-m_{\ell}^{-}$. In terms of these modes, Fermi surface deformations are parametrized as

$$
\begin{aligned}
& \delta n_{S}(\mathbf{q}, t)=m_{0}(\mathbf{q}, t)+ \\
& \sum_{\ell=1}^{\infty}\left\{m_{\ell}^{+}(\mathbf{q}, t) \cos \left(\ell \theta_{S}\right)+m_{\ell}^{-}(\mathbf{q}, t) \sin \left(\ell \theta_{S}\right)\right\} .
\end{aligned}
$$


Eliminating in equation (9) odd components in favor of even ones, we obtain the set of coupled differential equations $s^{7,28}$

$$
\begin{aligned}
& \frac{\partial^{2} m_{\ell}^{ \pm}(\mathbf{q}, t)}{\partial t^{2}}+\left(\frac{v_{F} q}{2}\right)^{2}\left[A_{\ell} m_{\ell}^{ \pm}(\mathbf{q}, t)+\right. \\
& \left.\quad+C_{\ell-1} m_{\ell-2}^{ \pm}(\mathbf{q}, t)+C_{\ell+1} m_{\ell+2}^{ \pm}(\mathbf{q}, t)\right]=0
\end{aligned}
$$

with dimensionless coefficients

$$
A_{\ell}=\alpha_{\ell}\left(\alpha_{\ell-1}+\alpha_{\ell+1}\right), C_{\ell}=\alpha_{\ell} \sqrt{\alpha_{\ell+1} \alpha_{\ell-1}} .
$$

The first line of Eq. (12), represents an harmonic oscillation for the mode $m_{\ell}^{ \pm}$with angular frequency $\omega^{2}=\left(v_{F} q / 2\right)^{2} A_{\ell}$. The second line couples the angular momentum mode $\ell$ with the corresponding modes $\ell+1$ and $\ell-1$. In this way, the model is mapped into a chain of harmonic oscillators with "first near neighbors" couplings. Due to parity symmetry, symmetric and antisymmetric modes $\left(m_{\ell}^{+}, m_{\ell}^{-}\right)$, as well as odd and even angular momentum modes, are completely decoupled. This structure enormously simplifies the analysis of the dynamics. The presence of a magnetic field breaks parity, mixing all modes in a nontrivial way $29-31$.

\section{MODEL FOR DENSITY AND QUADRUPOLAR INTERACTIONS}

The Landau theory of a Fermi liquids can be considered as a fixed point in the renormalization group sence. In particular, it was shown $\frac{14,32,33}{}$ that forward scattering interactions labeled by Landau parameters, $F_{\ell}$, with $\ell=0,1,2,3, \ldots$ are marginal. This result was criticized ${ }^{34}$ based on the fact that small angle singularities could generate, in the renormalization group flux, higher Landau harmonics, invalidating, in some sense, simple models with few Landau parameters. Some aspects of this idea were recently applied to dipolar Fermi gases 35 . The effect of small angle singularities could be specially dangerous near Pomeranchuk instabilities, however it seems to be irrelevant in the Fermi liquid phase at reasonable weak coupling. With this caveat in mind, and since we are interested in studying quadrupolar interactions in the stable Fermi liquid phase, we choose a Fermi liquid model whose interactions are parametrized by Landau parameters $F_{0}$ and $F_{2}$. Thus, we ignore any other component of the interaction, $F_{\ell}=0$ for $\ell \neq 0,2$. Then, the system of Eq. (12) is characterized by two parameters $\alpha_{0}=1+F_{0}$ and $\alpha_{2}=1+F_{2}$, with $\alpha_{\ell}=1$ for all $\ell \neq 0,2$.

Since odd and even modes are decoupled, let us focus on even angular momentum modes, $m_{0}^{ \pm}(\mathbf{q}, t), m_{2}^{ \pm}(\mathbf{q}, t)$, $m_{4}^{ \pm}(\mathbf{q}, t), \ldots$. By arranging these modes in a vector form $\mathbf{m}^{ \pm}=\left(m_{0}^{ \pm}, m_{2}^{ \pm}, \ldots\right)$, we can rewrite Eq. (12), in matrix notation. Fourier transforming in time and defining the dimensionless variable $s=\omega / v_{F} q$, where $\omega$ is the frequency, we have

$$
\left(s^{2} \mathbf{I}-\mathbf{M}^{ \pm}\right) \mathbf{m}^{ \pm}=0,
$$

where $\mathbf{I}$ is the identity matrix and $\mathbf{M}^{ \pm}$are two independent matrices driving the dynamics of the symmetric modes $\left(\mathbf{M}^{+}\right)$ and the antisymmetric ones $\left(\mathbf{M}^{-}\right)$.
We want to compute the Green functions

$$
\mathbf{G}^{ \pm}(\omega, \mathbf{q})=\left(s^{2} \mathbf{I}-\mathbf{M}^{ \pm}\right)^{-1} .
$$

Explicitly, we have

$$
\left[\mathbf{G}^{+}\right]^{-1}=\left(\begin{array}{cc|ccc}
s^{2}-\frac{\alpha_{0}}{2} & -\frac{\sqrt{\alpha_{0} \alpha_{2}}}{2} & 0 & 0 & \cdots \\
-\frac{\sqrt{\alpha_{0} \alpha_{2}}}{4} & s^{2}-\frac{\alpha_{2}}{2} & -\frac{\sqrt{\alpha_{2}}}{4} & 0 & \\
\hline 0 & -\frac{\sqrt{\alpha_{2}}}{4} & s^{2}-\frac{1}{2} & -\frac{1}{4} & \cdots \\
0 & 0 & -\frac{1}{4} & s^{2}-\frac{1}{2} & \\
\vdots & & \vdots & & \ddots
\end{array}\right)
$$

for the symmetric modes, and

$$
\left[\mathbf{G}^{-}\right]^{-1}=\left(\begin{array}{c|ccc}
\frac{s^{2}-\frac{\alpha_{2}}{2}}{\alpha^{2}} & -\frac{\sqrt{\alpha_{2}}}{4} & 0 & \ldots \\
\hline-\frac{\sqrt{\alpha_{2}}}{4} & s^{2}-\frac{1}{2} & -\frac{1}{4} & \cdots \\
0 & -\frac{1}{4} & s^{2}-\frac{1}{2} & \\
\vdots & \vdots & & \ddots
\end{array}\right)
$$

for the antisymmetric ones. Notice that in the antisymmetric channel there is no isotropic mode $m_{0}^{-} \equiv 0$. For this reason, while the first matrix acts on the space spanned by $\left(m_{0}, m_{2}^{+}, m_{4}^{+}, \ldots\right)$, the second matrix acts on the space spanned by $\left(m_{2}^{-}, m_{4}^{-}, \ldots\right)$. For the same reason, the coupling constant $\alpha_{0}$ does not affect the dynamics of the antisymmetric modes. We have explicitly indicated the block structure of Eqs. (16) and (17) by means of auxiliary vertical and horizontal lines inside the matrices. The first quadrant of the matrix $\left[\mathbf{G}^{+}\right]^{-1}$ is a $2 \times 2$ block, containing essentially the couplings of the modes $m_{0}$ and $m_{2}^{+}$. The fourth quadrant, in principle a block with infinite components, has no coupling constant and it is a tridiagonal matrix, representing the remaining "free" higher angular momentum modes. In this sense, the higher angular momentum modes are acting as a heat bath for the isotropic and quadrupole modes. Both blocks are coupled just by one element which is out of diagonal blocks, by means of the quadrupolar interaction $\alpha_{2}$. Eq. (17) has a similar structure, indeed a simpler one, since there is no isotropic coupling $\alpha_{0}$.

\section{GREEN FUNCTIONS}

Let us focus on the computation of some elements of the Green functions $\mathbf{G}^{ \pm}(s, \mathbf{q})$. We are particularly interested in the first $2 \times 2$ block

$$
\overline{\mathbf{G}}^{ \pm}(\omega, \mathbf{q})=\left(\begin{array}{ll}
G_{00}^{+}(\omega, \mathbf{q}) & G_{02}^{+}(\omega, \mathbf{q}) \\
G_{20}^{+}(\omega, \mathbf{q}) & G_{22}^{ \pm}(\omega, \mathbf{q})
\end{array}\right) .
$$

Notice that $G_{00}^{-}=G_{02}^{-}=G_{20}^{-}=0$, since there are no antisymmetric isotropic mode $m_{0}^{-} \equiv 0$. Thus, the Green function for the antisymmetric mode $m_{2}^{-}$is the single function $G_{22}^{-}$. With these Green functions at hand, it is possible to study time evolution of the modes $m_{2}^{ \pm}(t)$ and $m_{0}(t)$, provided we establish initial conditions in some of these two channels. 
To compute $\mathbf{G}^{ \pm}$, we use a recursive decimation procedure which is an application of the real space renormalization group theory to a one-dimensional chain ${ }^{20-23}$. The method is conceptually simple. We begin by truncating the matrices of Eqs. (16) and (17) to a finite range $n$. We invert the finite range matrix finding a recursive relation of the type

$$
\mathbf{G}^{ \pm(n+1)}=\mathcal{F}\left[\mathbf{G}^{ \pm(n)}\right]
$$

where $\mathbf{G}^{ \pm(n)}$ is the inverse of Eqs. (16) and 17), respectively, truncated to order $n . \mathcal{F}$ is some matrix function. The exact solution is found by taking the limit $\overline{\mathbf{G}}^{ \pm}=\lim _{n \rightarrow \infty} \mathbf{G}^{ \pm(n)}$. In Appendix A we show the explicit computation in detail. We find the following results,

$$
\overline{\mathbf{G}}^{+}(s, q)=\frac{1}{D^{+}(s, q)} \mathbf{N}^{+}(s, q)
$$

where the numerator

$$
\mathbf{N}^{+}(s, q)=\left(\begin{array}{cc}
s^{2}-\alpha_{2}\left(\frac{1}{2}+\Pi(s)\right) & \frac{\sqrt{\alpha_{0} \alpha_{2}}}{2} \\
\frac{\sqrt{\alpha_{0} \alpha_{2}}}{4} & s^{2}-\frac{\alpha_{0}}{2}
\end{array}\right)
$$

and the denominator

$$
D^{+}(s, q)=\left[s^{2}-\alpha_{2}\left(\frac{1}{2}+\Pi(s)\right)\right]\left(s^{2}-\frac{\alpha_{0}}{2}\right)-\frac{\alpha_{2} \alpha_{0}}{8} .
$$

For the antisymmetric modes, we find similar results

$$
\bar{G}^{-}(s, q)=\frac{s^{2}-\frac{1}{2}-\Pi(s)}{D^{-}(s, q)}
$$

with

$$
D^{-}(s, q)=\left[s^{2}-\frac{1}{2}-\Pi(s)\right]\left(s^{2}-\frac{\alpha_{2}}{2}\right)-\frac{\alpha_{2}}{16} .
$$

In Eqs. 21, 22, 23) and 24)

$$
\Pi(s)= \begin{cases}\frac{1}{2}\left\{s^{2}-\frac{1}{2} \pm|s| \sqrt{s^{2}-1}\right\} & \text { for } s>1 \\ \frac{1}{2}\left\{s^{2}-\frac{1}{2} \pm i|s| \sqrt{1-s^{2}}\right\} & \text { for } s<1\end{cases}
$$

Please, see Appendix A for its derivation.

The physical interpretation of this result is very interesting. We are computing the Green functions for the modes $m_{0}, m_{2}^{ \pm}$, taking into account their interactions with a "heat bath" composed by all other angular momentum modes. It is worth to note that the Green functions truncated to order $n$ (see Appendix A have $2 n$ real poles in the region $-1<s<1$. In the limit $n \rightarrow \infty$, the poles are dense, giving rise to a cut for $s^{2}<1$, as can be seen in Eq. (25). Moreover, for $s^{2}>1$, $\Pi(s)$ is real, while for $s^{2}<1$, it gets an imaginary part. This is nothing but Landau damping. In next sections we explicitly compute collective modes and spectral functions.

\section{COLLECTIVE MODES}

In usual Fermi liquids with isotropic interactions, there are only symmetric collective modes. However, when higher Landau parameters are considered, antisymmetric modes are also possible. In the following, we analyze these two cases separately.

\section{A. Symmetric collective modes}

In order to compute symmetric collective modes, we look for solutions of $D^{+}(s)=0$, where $D^{+}(s)$ is given by Eq. 22 with $\alpha_{0}>0$ and $\alpha_{2}>0$. $\Pi(s)$ is given by Eq. 25.

The first thing to note is that $D^{+}(0)=0$ independently of the interactions $\alpha_{0}$ or $\alpha_{2}$. This zero mode is associated with static local reparametrization of the Fermi surface and, thus, it is not physical. In this section, we will seek solutions with $s \neq$ 0 . Later, when computing spectral functions, we will analyze this "spurious" zero mode in detail. To check the consistency of the method, let us look for solutions in the simplest case of $\alpha_{2}=1$, i.e., a system with only density interactions $\alpha_{0}=$ $1+F_{0}$. We easily find the single solution

$$
s^{2}=\frac{\alpha_{0}^{2}}{2 \alpha_{0}-1}=\frac{\left(1+F_{0}\right)^{2}}{1+2 F_{0}},
$$

which is the well-known Landau zero sound in two dimensions 28 .

Equivalent collective modes in the case of pure quadrupolar interactions, i.e., $\alpha_{0}=1$ and $\alpha_{2} \neq 1$, can be found. In this case, $D^{+}(s)=0$ reduces to a cubic polynomial equation in $s^{2}$. We depicted the roots in Fig. 1 as a function of the quadrupolar interaction $\alpha_{2}$.

In panel 1(a), we show the real part of the roots, while in panel 1(b) we depict the corresponding imaginary part. We can observe an interesting structure. For repulsive interactions, $\alpha_{2}>1$, the system has one real positive root with $s^{2}>1$. This is the analog of the Landau zero sound for the quadrupolar interaction. We can give explicit analytic expressions for weak as well as strong repulsion,

$$
s^{2}=\left\{\begin{array}{lll}
1+4\left(\alpha_{2}-1\right)^{2}+\ldots & \text { for } & \alpha_{2} \gtrsim 1 \\
\frac{3}{8}+\frac{1}{2} \alpha_{2}+O\left(1 / \alpha_{2}\right) & \text { for } & \alpha_{2} \gg 1
\end{array}\right.
$$

Moreover, we have one additional complex root (and its complex conjugate) representing damped modes,

$$
s^{2} \sim \begin{cases}\frac{1}{4} \pm i \frac{1}{4 \sqrt{\alpha_{2}-1}} & \text { for } \quad \alpha_{2} \gtrsim 1 \\ \frac{5}{16} \pm \frac{4}{25} i+O\left(1 / \alpha_{2}\right) & \text { for } \quad \alpha_{2} \gg 1\end{cases}
$$

This mode is inside the particle-hole continuum of the spectrum. Very near the Fermi gas, when $\alpha_{2} \rightarrow 1^{+}$, the real root $s^{2} \rightarrow 1$ and the imaginary part of the complex one diverges, since in the free case only the mode $s^{2}=1$ should survive.

The attractive quadrupolar case is more interesting. In this case, there is an overdamped mode in all the attractive regime 


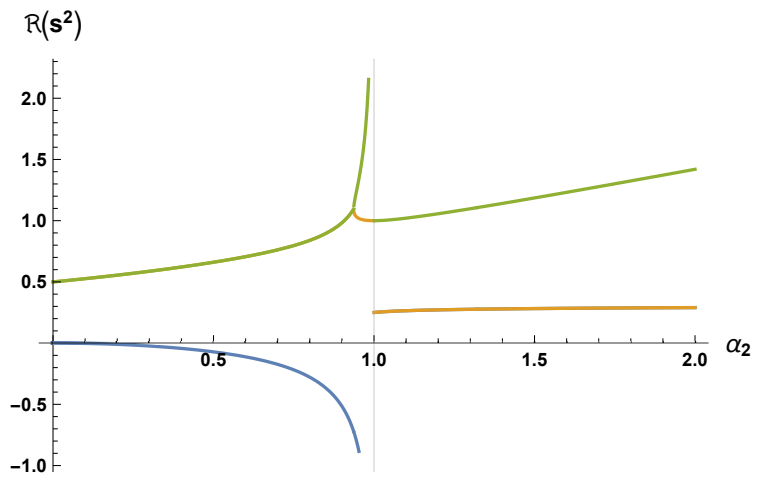

(a)

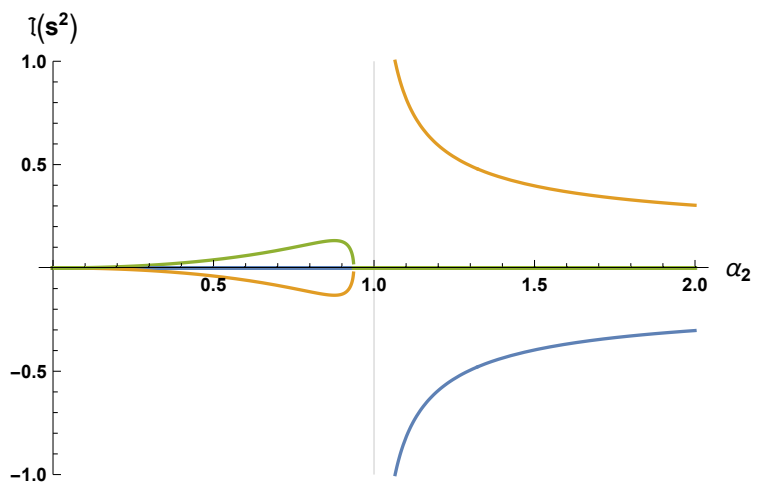

(b)

FIG. 1. Symmetric collective modes. Solutions of the equation $D^{+}(s)=0$, with $D^{+}(s)$ given by Eq. (22). In panel 1(a) we plot $\Re\left[s^{2}\left(\alpha_{2}\right)\right]$ while in panel[1(b) we depict $\Im\left[s^{2}\left(\alpha_{2}\right)\right]$. In both panels we have fixed $\alpha_{0}=1$.

$\left(0<\alpha_{2}<1\right)$. We have,

$$
s^{2}= \begin{cases}-\frac{1}{4} \frac{1}{\sqrt{1-\alpha_{2}}} & \text { for } \alpha_{2} \lesssim 1 \\ -\frac{\alpha_{2}^{2}}{4} & \text { for } \alpha_{2} \gtrsim 0\end{cases}
$$

In addition, for weak attraction, $\alpha_{2} \lesssim 1$, there are two stable collective modes, well separated from the particle-hole continuum. They take the form,

$$
\begin{aligned}
& s_{1}^{2} \sim 1+\frac{19}{4}\left(1-\alpha_{2}\right)+O\left(\left(1-\alpha_{2}\right)^{2}\right), \\
& s_{2}^{2} \sim \frac{1}{8} \frac{1}{\sqrt{1-\alpha_{2}}}+O\left(\left(1-\alpha_{2}\right)^{-3 / 2}\right) .
\end{aligned}
$$

$s_{1}$ is the continuation of the quadrupolar zero sound observed in the repulsive case to the weak attractive regime. On the other hand, $s_{2}$ is proper of attractive interactions and it has no counterpart in the repulsive regime. For moderate attractions both stable modes meet each other, merging in a damped mode that remains in the strong attractive limit. Thus, we clearly see two points where the dynamical regime changes abruptly. One point is $\alpha_{2}=1$, the transition between the repulsive and attractive regime. The other point, $\alpha_{2 c} \sim 0.93$, is when two real poles transform in a double pole acquiring an imaginary part for $\alpha_{2}<\alpha_{2 c}$. To the best of our knowledge, this is an unexpected feature and is an example of an "exceptional point", studied in the literature of non-hermitian effective Hamiltonians 26 .

Repulsive isotropic interactions, $\alpha_{0}>1$, do not change the root structure. Indeed, the only effect is to increase the region with two stable modes. On the contrary, attractive interactions, $\alpha_{0}<1$, reduce this region that eventually disappears. These stable modes reappear for strong enough attractive isotropic interactions. This feature is depicted in Fig. (2). Here, we show a dynamical phase diagram in the $\alpha_{2}-\alpha_{0}$ plane. The white area represent the region where two real roots separated from the particle-hole continuum are present. The border lines of these regions indicate a sudden change in the dynamical properties of the system signaling a dynamical phase transition. Indeed, the transition line in the attractive region has a double pole in the Green functions, producing linear running out modes in real time.

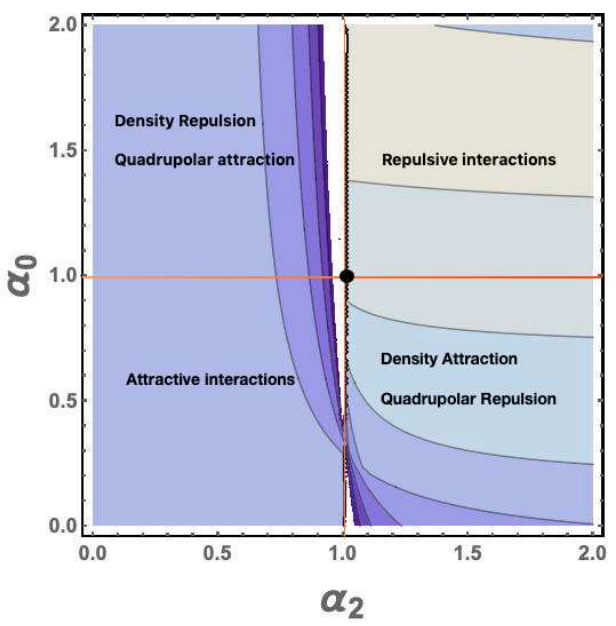

FIG. 2. Dynamical phase diagram in the $\alpha_{2}-\alpha_{0}$ plane, with $\alpha_{0}=$ $1+F_{0}$ and $\alpha_{2}=1+F_{2}$. The central bold point $\alpha_{0}=\alpha_{2}=$ 1 correspond to the Fermi Gas. $\alpha_{0,2}>1\left(\alpha_{0,2}<1\right)$ represent repulsive (attractive) interactions. The white region is the one where two stable collective modes are present. In the borders of this region there is sharp change in the collective modes structure, signaling a dynamical phase transition.

\section{B. Antisymmetric modes}

In order to look for antisymmetric modes, we need to solve $D^{-}(s)=0$, where $D^{-}(s)$ is given by Eq. (24). Note that this expression is $\alpha_{0}$ independent. We find the solutions

$$
s^{2}=\frac{\alpha_{2}}{4}\left\{\begin{array}{l}
1 \pm \sqrt{\frac{\alpha_{2}}{\alpha_{2}-1}} \text { for } \alpha_{2}>1 \\
1 \pm i \sqrt{\frac{\alpha_{2}}{1-\alpha_{2}}} \text { for } \alpha_{2}<1
\end{array}\right.
$$

which are depicted in Fig. (3). For repulsive quadrupolar interaction $\left(\alpha_{2}>1\right)$, we have an overdamped and a stable mode. These modes are not possible without higher angular 


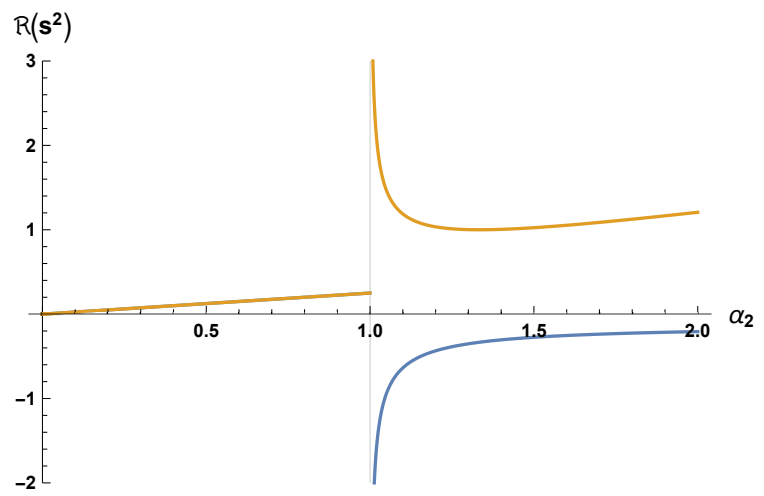

(a)



(b)

FIG. 3. Antisymmetric collective modes. Solutions of the equation $D^{-}(s)=0$ with $D^{-}(s)$ given by Eq. 24. In panel 3(a) we plot $\Re\left[s^{2}\left(\alpha_{2}\right)\right]$ while in panel $3(\mathrm{~b})$ we depict $\Im\left[s^{2}\left(\alpha_{2}\right)\right]$. In both panels we have fixed $\alpha_{0}=1$.

momentum interactions. Note that both roots diverges in the limit $\alpha_{2} \rightarrow 1$. In the attractive region, there is a damped mode that runs all the way to the Pomeranchuk instability. In next section, we study in detail this region.

\section{Pomeranchuk regime}

The model described so far is reliable provided $\alpha_{0}>0$ and $\alpha_{2}>0$. Exactly at the values $\alpha_{0}=0$ or $\alpha_{2}=0$, the theory is unstable and the harmonic approximation is no longer valid. These instabilities are known as Pomeranchuk instabilities. The $\alpha_{2}=0$ Pomeranchuk instability is associated with the quantum critical isotropic-nematic phase transition ${ }^{2,7}$. Therefore, in order to understand this transition it is useful to know the structure of collective modes near $\alpha_{2} \gtrsim 0$. In our formalism, the instability is manifested by the appearance of a mode $s^{2}=0$ at $\alpha_{2}=0$. However, this is because we have chosen a model with highly localized interactions. In fact, in Eq. (4), we have considered $F_{S, T}\left(\mathbf{x}-\mathbf{x}^{\prime}\right) \equiv F_{S, T} \delta^{2}\left(\mathbf{x}-\mathbf{x}^{\prime}\right)$. It leads to constant parameters $\alpha_{0}, \alpha_{2}$. This model is not adequate near a Pomeranchuk instability and we need to consider finite ranged interactions. In this way, the parameters of the model are momentum dependent, i.e., $\alpha_{0} \equiv \alpha_{0}(q)$ and $\alpha_{2} \equiv \alpha_{2}(q)$.
For short range interactions, it is possible to expand these couplings in Taylor series,

$$
\begin{aligned}
& \alpha_{0}(q)=\alpha_{0}+\kappa_{0} q^{2} \\
& \alpha_{2}(q)=\alpha_{0}+\kappa_{2} q^{2}
\end{aligned}
$$

where $\kappa_{0}^{2}$ and $\kappa_{2}^{2}$ are typical short distance ranges for density and quadrupolar interactions, respectively. Deep in the Fermi liquid phase, these momentum dependent functions are irrelevant and it is sufficient to consider the zero momentum constant Landau parameters. However, near Pomeranchuk instabilities, the momentum dependent terms are important. Expanding the symmetric as well as the antisymmetric collective modes in powers of $\alpha_{2}(q) \gtrsim 0$ and setting the Pomeranchuk condition $\alpha_{2}=0$, we find,

$$
\begin{array}{lll}
s^{2} \sim \frac{\alpha_{0}}{2} & \rightarrow \omega \sim \sqrt{\frac{\alpha_{0}}{2}} v_{F} q & \text { symmetric } \\
s^{2} \sim-\frac{\alpha_{2}^{2}(q)}{4} \rightarrow \omega \sim i \frac{1}{2} \kappa_{2} v_{F} q^{3} & \text { symmetric } \\
s^{2} \sim \frac{\alpha_{2}(q)}{4} \rightarrow \omega \sim \frac{1}{2} \sqrt{\kappa_{2}} v_{F} q^{2} & \text { antisymmetric }
\end{array}
$$

We see that we have a stable linear mode, Eq. (35), and an overdamped cubic mode, Eq. (36), associated with the symmetric collective modes. In addition, we have a stable quadratic mode, Eq. 37, associated with antisymmetric collective modes. The overdamped cubic mode was identified as the most relevant to describe the isotropic-nematic phase transition, having its counterpart on the broken symmetry phase as a Goldstone mode ${ }^{7}$.

\section{SPECTRAL FUNCTIONS}

As already mentioned in the previous section, the Green function for the symmetric sector diverges as $s \rightarrow 0$. The reason is the existence of a zero eigenvalue of the matrix $\mathbf{M}^{+}$ in Eq. (14). The existence of this zero mode does not depend on interactions, it is present even in the Fermi gas case, $\alpha_{0}=\alpha_{2}=1$. To understand the physical meaning of this mode, it is convenient to turn back to the original equation, Eq. (6). For static deformations, in the free case, we have $\left(\mathbf{v}_{S} \cdot \mathbf{q}\right) \delta n_{S}(\mathbf{q}, t)=0$. If $\left(\mathbf{v}_{S} \cdot \mathbf{q}\right) \neq 0$ then, the only static solution is $\delta n_{S}(\mathbf{q})=0$ for all $S$. However, if $\left(\mathbf{v}_{S} \cdot \mathbf{q}\right)=0$, then there is a nontrivial solution $\delta n_{S}(\mathbf{q})=$ constant, with $\mathbf{q} \perp \mathbf{v}_{S}$. In this way, constant displacements perpendicular to Fermi velocity in each patch are solutions of the equation of motion. However, tangential displacements do not deform the Fermi surface, they are just reparametrizations of the same surface. It is instructive to understand this result in the angular momentum basis. From Eq. (9), it is simple to see that in the free case, there are nontrivial static solutions of the form $m_{\ell-1}=-m_{\ell+1}$. This means, for instance, that $m_{0}=-m_{ \pm 2}=m_{ \pm 4}=-m_{ \pm 6}, \ldots$ are nontrivial solutions of the equation $\mathbf{M}^{+} \mathbf{m}^{+}=0$. Thus, the vector $\mathbf{m}^{+}=m_{0}(1,-1,1,-1, \ldots)$ is an eigenvector of $\mathbf{M}$ with zero eigenvalue. To understand the meaning of this eigenvector, we 
can replace it in Eq. (7). We find,

$$
\delta n_{S}(\mathbf{q})=m_{0} \sum_{\ell=-\infty}^{\infty}(-1)^{\ell} e^{i 2 \ell \theta_{S}}=m_{0} \delta\left(\theta_{S}-\frac{\pi}{2}\right) .
$$

Thus, the zero mode represent particle-hole excitations with momentum q perpendicular to the Fermi velocity in each patch. It is simple to solve this problem in the angular momentum basis. The key observation is that, since the eigenvectors of the zero mode do not represent actual physical excitations, the physical vector space is the orthogonal subspace to the zero mode eigenvector. Thus, we need to compute the modified Green functions by restricting the vector space. This process is practically simple to implement. We need to expand the Green function in partial fractions and define the modified Green functions by simply subtracting the contribution from the zero mode. Then, the modified Green function takes the general form

$$
\mathbf{G}_{R}(s, q)=\sum_{j} \frac{B_{j}(s, q)}{\left(s^{2}-s_{j}^{2}\right)}
$$

where $s_{j}^{2} \neq 0$ are the nontrivial roots computed in the previous section and the weights $B_{j}(s, q)$ are simply computed by expanding the function in partial fractions.

We computed the normalized spectral functions as

$$
\mathbf{A}^{ \pm}(\omega, \mathbf{q})=\mathcal{N}^{-1} \lim _{\epsilon \rightarrow 0^{+}} \Im\left[\mathbf{G}_{\mathbf{R}}^{ \pm}(\omega+i \epsilon, q)\right]
$$

where the normalization constant $\mathcal{N}^{-1}$ is chosen in such a way that $\int_{-\infty}^{+\infty} \mathbf{A}(\omega, \mathbf{q}) d \omega=1$ for fixed $\vec{q}$. In Figure 4, we show $A_{22}^{+}(s, \mathbf{q})$ for $\alpha_{0}=1$ and different values of the quadrupolar coupling $\alpha_{2}$. In panel 4(a) we fixed $\alpha_{2}=2$, deep in the repulsive region. $\operatorname{In} 4(\mathrm{~b})$, we show the spectral function for weak attraction $\alpha_{2}=0.94$, while the last panel 4(c) is in the strong repulsive regime, near the Pomeranchuk nematic instability, $\alpha_{2}=0.1$. In Fig. 4(a), we observe the quadrupolar Landau zero sound as a sharp peak in the repulsive quadrupolar regime. Moreover, we observe a continuum particle-hole distribution for $s<1$. This resonance moves to the left with decreasing $\alpha_{2}$ and reaches the free mode $s^{2}=1$ for $\alpha_{2}=1$. Figure 4(b) shows an unexpected result. In the attractive region, there are two resonances. The resonance near $s \gtrsim 1$ is the continuation of the zero sound to the attractive region. However, there is a second mode, beginning at very high frequency that moves to lower frequency for more attractive interactions. This behavior can be clearly observed in Fig. 1. Both resonances meet each other at a critical $\alpha_{2 c} \sim 0.93$, where there is a second order pole in the Green function. The location of this critical value depends on $\alpha_{0}$ and it is shown in Fig. 2. For smaller values of $\alpha_{2}$, the mode becomes damped and there is a huge transfer of spectral weight to the overdamped cubic mode, very near the Pomeranchuk instability.

We have also plotted in Fig. 5, the spectral function for the antisymmetric mode $A_{22}^{-}(s, q)$. Usually, in a Fermi liquid with isotropic interactions $F_{0}$, there are no sharp antisymmetric modes. However, they are possible when higher

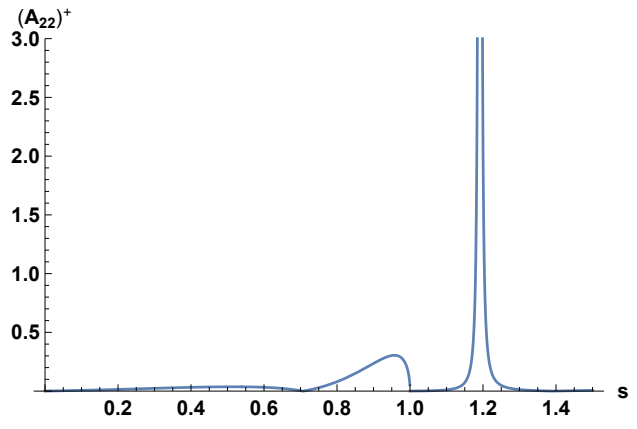

(a)

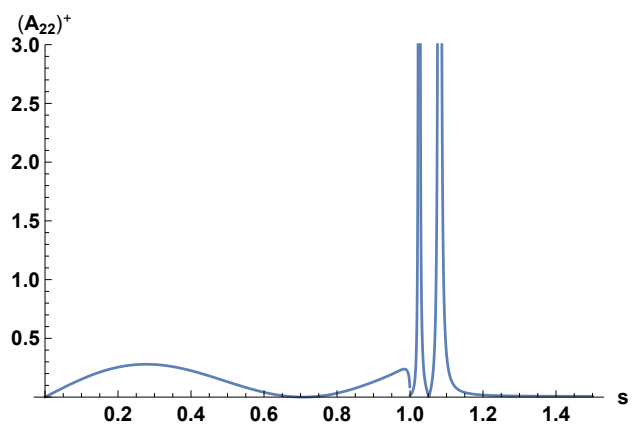

(b)

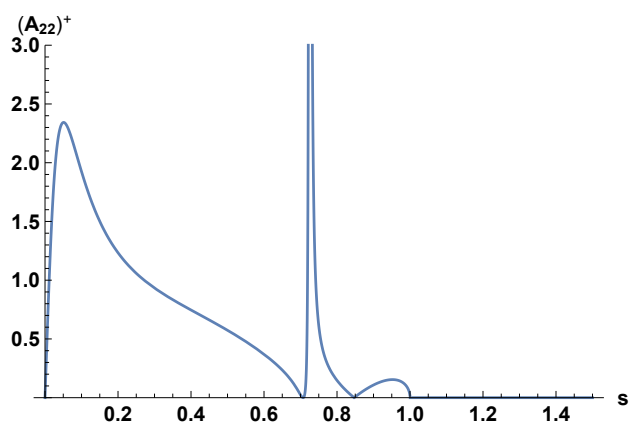

(c)

FIG. 4. Normalized spectral function $A_{22}^{+}$as a function of $s=$ $\omega / v_{F} q$, for $q$ fixed. We have fixed $\alpha_{0}=1$, in such a way that the roots coincide with those of Fig. 1 In 4(a) we have fixed $\alpha_{2}=2$, deep in the repulsive region. 4(b) is in the weak attractive region $\alpha_{2}=0.94$ (white region of Fig. 2), while in 4(c) we have fixed $\alpha_{2}=0.1$ near the Pomeranchuk instability.

angular momentum interactions are considered. Antisymmetric modes were computed for Fermi liquis with interactions $F_{0}$ and $F_{1} \frac{27}{2}$ and at the nematic Pomeranchuk instability ${ }^{\frac{7}{7}}$. Here, we show that the same type of mode also appears with quadrupolar interactions in all the interaction regime. However, for very strong repulsive interactions, the spectral weight of the share mode is very small, compared with the continuum particle-hole distribution, Fig. 5(a). For small repulsion $\alpha_{2}=1.5$, this mode is sharply defined (see Fig. 5(b) and is well separated from the particle-hole continuum for very weak repulsive interactions. For attractive interactions, this mode becomes damped, and very near the Pomeranchuk instability, this gets most of the spectral weight near $s \sim 0$, producing a well defined mode with a quadratic dispersion relation. 


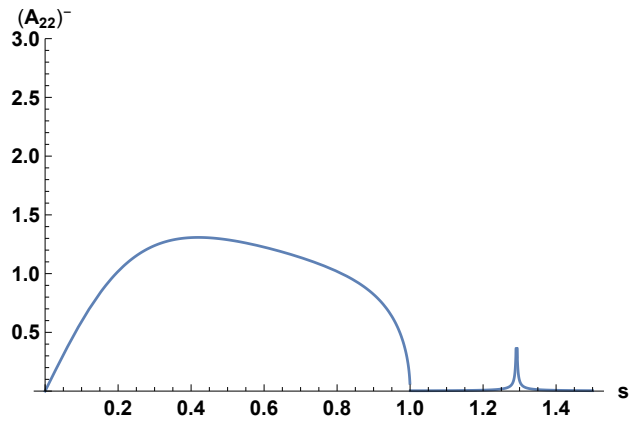

(a)

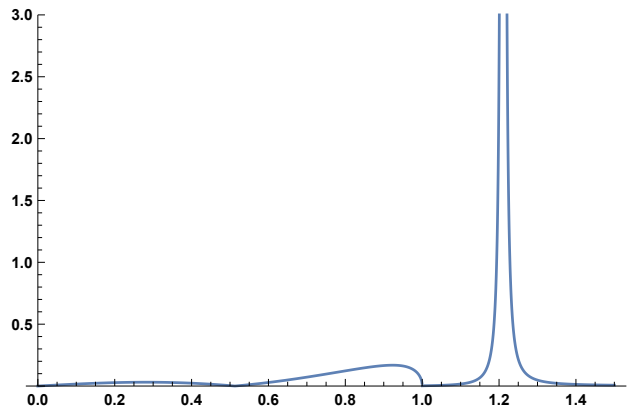

(b)

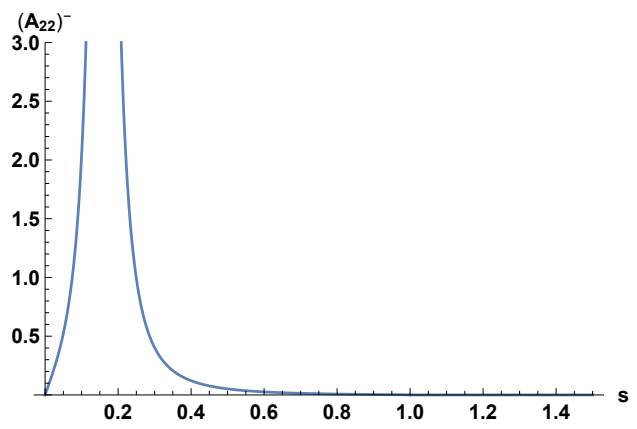

(c)

FIG. 5. Normalized spectral function $A_{22}^{-}$as a function of $s=$ $\omega / v_{F} q$, for $q$ fixed. The roots coincide with those of Fig. 3 In 5(a) we have fixed $\alpha_{2}=2$, deep in the repulsive region. Fig. 5(b) is computed for weak repulsion $\alpha_{2}=1.5$, while in 4(c) we have fixed $\alpha_{2}=0.1$ near the Pomeranchuk instability.

\section{SUMMARY AND DISCUSSIONS}

We have presented a detailed study of collective modes of a Fermi liquid model with density and quadrupolar interactions. We have analyzed a wide range of interactions, from a repulsive regime to a strong attractive region in both interaction channels. We have used a bosonization approach to Fermi liquids to deduce a semiclassical evolution equation for Fermi surface fluctuations parametrized by an infinite set of angular momentum modes. In this way, the system is mapped into a one-dimensional chain of angular momentum modes with first near neighbors interactions. Due to parity symmetry, longitudinal (symmetric) and transverse (antisymmetric) modes are decoupled. Moreover, odd and even angular momentum excitations are also decoupled. By means of a recurrent decimation procedure, we have computed the relevant Green functions. Exploring the analytic properties, we were able to study collective modes and spectral functions for different values of the interaction parameters $\alpha_{0}=1+F_{0}$ and $\alpha_{2}=1+F_{2}$.

In the repulsive region $\left(\alpha_{0}>1, \alpha_{2}>1\right)$, we have found the expected Landau zero sound excitations, modified by the presence of quadrupolar interactions. For antisymmetric (transverse) modes, we have also find a stable mode, compatible with a "share sound" recently reported in a similar related system $^{27}$. However, we found that the spectral weight of this mode is highly suppressed for strong repulsion, having a clear signature above the particle-hole continuum for very weak repulsion, very near the Fermi gas region. It is important to note, that antisymmetric modes are not possible in Fermi liquids with only isotropic interactions $\alpha_{0}$.

When attractive interactions are present $\left(\alpha_{0}<1\right.$ or/and $\alpha_{2}<1$ ), the dynamical structure is more interesting. We found that weak quadrupolar attraction $\left(\alpha_{0}=1, \alpha_{2} \lesssim 1\right)$ produces a sudden change in the symmetric modes dynamics. In addition with the continuation of the zero sound excitation, there is another stable mode at very high frequencies which moves to lower frequencies when the attraction growths. There is a critical value $\alpha_{2 c}$, in which both stable modes meet together, melting in a single damped mode for stronger attraction $\left(\alpha_{2}<\alpha_{2 c}\right)$. Exactly at $\alpha_{2 c}$, there is a double pole in the Green function, producing a linear running out mode in real time. For stronger attraction, besides the damped mode, an overdamped one gains spectral weight, being the dominant mode near the Pomeranchuk region $\alpha_{2} \gtrsim 0$. This behavior is sketched in Fig. (4). This general collective modes structure remains the same in the presence of repulsive isotropic interactions $\alpha_{0}>1$. The effect of this coupling is to enlarge the intermediate region with two stable modes. However, attractive isotropic couplings $\alpha_{0}<1$ shrink this region which eventually disappears. Interestingly, an equivalent behavior is observed in a dual region $\alpha_{0}<1$ with $\alpha_{2}>1$, and remains unchanged up to the Pomeranchuk region $\alpha_{0} \gtrsim 0$. We have illustrated this behavior in a dynamical phase diagram in Fig. 2. In this diagram, we show the lines where the structure of collective modes changes abruptly, possibly signaling a dynamical phase transition. The physics behind this transition can be traced back to the fact that the model behaves like a small quantum system, containing few degrees of freedom (an isotropic "breathing mode" and a quadrupolar "nematic" excitation), in contact with a non-trivial environment composed by an infinite set of angular momentum excitations. The coupling between the small effective system and the environment is governed by $\sqrt{\alpha_{2}}$. The effective Hamiltonian is non-hermitian, signaling a dissipative behavior of the reduced system. It is interesting to note that some small dissipative quantum systems present, depending on the environment, dynamical phase transitions, characterized by crossing of energy levels, where some properties of the eigen-functions such as orthogonality and phase rigidity dramatically change ${ }^{26}$. The term "dynamical phase transition" is also used in another context to denote a lack of analyticity in some time-dependent self-correlation functions. This point of view is usually ap- 
plied in the study of closed system, where the time evolution is unitary. There should be a deep relation between these two approaches. However, to the best of our knowledge, this issue is not completely understood so far ${ }^{36}$.

Summarizing, the main novel features we present in this paper are, in one hand, the observation of a sudden change in the collective mode structure which appears when the system mixes attractive and repulsive isotropic and quadrupolar interactions. On the other hand, we show the existence of a sharp antisymmetric mode, very well defined for weak quadrupolar repulsion.

The discotinuos dynamical behavior described in the paper will have an impact on the out-of-equilibirum Fermi surface evolution in quantum quenches set ups 37 . Although we have presented a simple phenomenological model, we believe that our results could also help to understand non-equilibrium responses of different systems that are being explored experimentally, especially those in which quadrupolar interactions seem to be relevant. Iron based superconductors are promising compounds 38,39 , which have clear evidences of electronic nematic phases. Moreover, quantum Hall systems at partially filled Landau levels also display spontaneous breakdown of rotational symmetry ${ }^{5,40}-\underline{42}$, signaling the relevance of the quadrupolar interaction. Since much of the interesting features of our model have signatures for weak interactions, we expect that ultracold Fermionic atoms ${ }^{43,44}$ could also be an arena to look for this phenomenology, due to the fact that weak repulsive as well as attractive couplings can be manipulated with great precision in these systems.

\section{ACKNOWLEDGMENTS}

We would like to acknowledge Eduardo Fradkin, Anibal Iucci and Zochil González Arenas for useful comments. The Brazilian agencies, Fundação de Amparo à Pesquisa do Rio de Janeiro (FAPERJ), Conselho Nacional de Desenvolvimento Científico e Tecnológico $(\mathrm{CNPq})$ and Coordenação de Aperfeiçoamento de Pessoal de Nivel Superior (CAPES) - Finance Code 001, are acknowledged for partial financial support. RA was partially supported by a MSc Fellowship from CAPES.

\section{Appendix A: Decimation procedure}

In order to compute the Green functions, Eqs. (16) and (17), we need to invert an infinite range matrix. The tridiagonal form of these matrices greatly simplifies the calculation. Indeed, the mathematical model described by these matrices are completely analog to a linear chain of harmonic oscillators. The angular momentum in our problem is playing the role of the "lattice site" in a tight-binding atomic chain. The decimation procedure is an effective algorithm to compute Green functions, specially in a one-dimensional lattice. The method can be summarized in the following way. The idea is to compute the inverse of a truncated matrix to order $n+1$, as a function of the inverse of the truncated matrix of order $n$. Provided this is possible, the exact Green function is computed by means of a recurrence relation in the limit of $n \rightarrow \infty$.

Consider, for instance, the matrix of Eq. (16). First, we truncate the matrix to order $n=2$. Then, we need to compute

$$
\left(\begin{array}{cc}
s^{2}-\frac{\alpha_{0}}{2} & \frac{\sqrt{\alpha_{0} \alpha_{2}}}{2} \\
\frac{\sqrt{\alpha_{0} \alpha_{2}}}{4} & s^{2}-\frac{\alpha_{2}}{2}
\end{array}\right)\left(\begin{array}{cc}
G_{00}^{+}(\omega, \mathbf{q}) & G_{02}^{+}(\omega, \mathbf{q}) \\
G_{20}^{+}(\omega, \mathbf{q}) & G_{22}^{+}(\omega, \mathbf{q})
\end{array}\right)=\mathbf{I}
$$

Let us focus in one component of the matrix, say $G_{00}^{+}$. Inverting the $2 \times 2$ matrix, we easily find,

$$
G_{00}^{+(2)}=\frac{1}{s^{2}-\frac{\alpha_{0}}{2}-\frac{\alpha_{0} \alpha_{2}}{8} \frac{1}{s^{2}-\frac{\alpha_{2}}{2}}}
$$

where the superscript (2) means that the matrix was truncated to order 2. Repeating the same procedure for truncations to order $n=3$ and $n=4$ we get, after direct calculation,

$$
\begin{aligned}
& G_{00}^{+(3)}=\frac{1}{s^{2}-\frac{\alpha_{0}}{2}-\frac{\alpha_{0} \alpha_{2}}{8} \frac{1}{s^{2}-\frac{\alpha_{2}}{2}-\frac{\alpha_{2}}{16} \frac{1}{s^{2}-\frac{1}{2}}}} \\
& G_{00}^{+(4)}= \\
& 1 \\
& \overline{s^{2}-\frac{\alpha_{0}}{2}-\frac{\alpha_{0} \alpha_{2}}{8} \frac{1}{s^{2}-\frac{\alpha_{2}}{2}-\frac{\alpha_{2}}{16} \frac{1}{s^{2}-\frac{1}{2}-\frac{1}{16} \frac{1}{s^{2}-\frac{1}{2}}}}}
\end{aligned}
$$

By carefully analyzing the recursive structure of $G_{00}^{+(2)}$, $G_{00}^{+(3)}, G_{00}^{+(4)}, \ldots$, we can write the $G_{00}^{+(n)}$ in the following way,

$$
G_{00}^{+(n)}=\frac{1}{s^{2}-\frac{\alpha_{0}}{2}-\frac{\alpha_{0} \alpha_{2}}{8} \frac{1}{s^{2}-\frac{\alpha_{2}}{2}-\alpha_{2} \Pi^{(n)}(s)}}
$$

where $\Pi^{(n)}(s)$ is given by the recurrence relation

$$
\Pi^{(n+1)}(s)=\frac{1}{16} \frac{1}{s^{2}-\frac{1}{2}-\Pi^{(n)}(s)}
$$

with the initial condition $\Pi^{(2)}(s)=0$.

The other components of the Green function, $G_{02}^{+}, G_{20}^{+}$and $G_{22}^{ \pm}$can be computed in the same way. We obtain for the symmetric modes

$$
\overline{\mathbf{G}}^{+(n)}(s)=\frac{1}{D^{+(n)}(s)} \mathbf{N}^{+(n)}(s)
$$


where the numerator

$$
\mathbf{N}^{+(n)}=\left(\begin{array}{cc}
s^{2}-\alpha_{2}\left(\frac{1}{2}+\Pi^{(n)}(s)\right) & \frac{\sqrt{\alpha_{0} \alpha_{2}}}{2} \\
\frac{\sqrt{\alpha_{0} \alpha_{2}}}{4} & s^{2}-\frac{\alpha_{0}}{2}
\end{array}\right)
$$

and the denominator

$$
D^{+(n)}=\left[s^{2}-\alpha_{2}\left(\frac{1}{2}+\Pi^{(n)}(s)\right)\right]\left(s^{2}-\frac{\alpha_{0}}{2}\right)-\frac{\alpha_{2} \alpha_{0}}{8} .
$$

Similarly, we obtain for the antisymmetric mode the Green function

$$
G_{22}^{-(n)}(s)=\frac{N^{-(n)}(s)}{D^{-(n)}(s)}
$$

where

$$
N^{-(n)}=s^{2}-\frac{1}{2}-\Pi^{(n)}(s)
$$

and

$$
D^{-(n)}=\left[s^{2}-\frac{1}{2}-\Pi^{(n)}(s)\right]\left(s^{2}-\frac{\alpha_{2}}{2}\right)-\frac{\alpha_{2}}{16} .
$$

Thus, we have inverted the matrices of Eqs. 16 and (17) by truncating them at any finite order $n$. The explicit expression for these matrices are given in terms of a recursion relation for the function $\Pi^{(n)}$, given by Eq. A6. To exactly compute the Green functions, we need to take the $n \rightarrow \infty$ limit. In that limit, $\Pi^{(n+1)}=\Pi^{(n)}$. Defining

$$
\Pi(s)=\lim _{n \rightarrow \infty} \Pi^{(n)}(s),
$$

we find, from Eq.

$$
\Pi(s)=\frac{1}{16} \frac{1}{s^{2}-\frac{1}{2}-\Pi(s)}
$$

which can be solved to get

$$
\Pi(s)=\left\{\begin{array}{cc}
\frac{1}{2}\left\{s^{2}-\frac{1}{2} \pm|s| \sqrt{s^{2}-1}\right\} & \text { for } s>1 \\
\frac{1}{2}\left\{s^{2}-\frac{1}{2} \pm i|s| \sqrt{1-s^{2}}\right\} & \text { for } s<1
\end{array}\right.
$$

Replacing $\Pi^{(n)}(s)$ by $\Pi(s)$ in Eqs. A8, A9, A11 and $\mathrm{A} 12$, we find the exact Green functions presented in Eqs. 201 to 25.
${ }^{1}$ E. Fradkin, S. A. Kivelson, M. J. Lawler, J. P. Eisenstein, and A. P. Mackenzie, Annual Review of Condensed Matter Physics 1, 153 (2010) http://www.annualreviews.org/doi/pdf/10.1146/annurev-conmat

2 V. Oganesyan, S. A. Kivelson, and E. Fradkin, Phys. Rev. B 64, 195109 (2001)

3 R. Daou, J. Chang, D. LeBoeuf, O. Cyr-Choiniere, F. Laliberte, N. Doiron-Leyraud, B. J. Ramshaw, R. Liang, D. A. Bonn, W. N. Hardy, and L. Taillefer, Nature 463, 519 (2010)

${ }^{4}$ M. A. Tanatar, A. E. Böhmer, E. I. Timmons, M. Schütt, G. Drachuck, V. Taufour, K. Kothapalli, A. Kreyssig, S. L. Bud'ko, P. C. Canfield, R. M. Fernandes, and R. Prozorov, Phys. Rev. Lett. 117, 127001 (2016)

5 E. Fradkin and S. A. Kivelson, Phys. Rev. B 59, 8065 (1999)

${ }^{6}$ R. A. Borzi, S. A. Grigera, J. Farrell, R. S. Perry, S. J. S. Lister, S. L. Lee, D. A. Tennant, Y. Maeno, and A. P. Mackenzie, Science 315, 214 (2007)

7 M. J. Lawler, D. G. Barci, V. Fernández, E. Fradkin, and L. Oxman, Phys. Rev. B 73, 085101 (2006).

${ }^{8}$ E. Berg, E. Fradkin, and S. A. Kivelson, Nature Physics 5, 830 EP (2009)

9 D. G. Barci and E. Fradkin, Phys. Rev. B 83, 100509 (2011)

10 D. G. Barci, R. V. Clarim, and N. L. Silva Júnior, Phys. Rev. B 94, 184507 (2016)

11 P. Nozieres and D. Pines, The Theory of Quantum Liquids (Perseus Books, 1999) p. 48.

12 A. H. Castro Neto and E. Fradkin, Phys. Rev. Lett. 72, 1393 (1994)

13 A. H. Castro Neto and E. Fradkin, Phys. Rev. B 49, 10877 (1994)

14 A. H. Castro Neto and E. H. Fradkin,
Phys. Rev. B 51, 4084 (1995)

15 A. Houghton and J. B. Marston, Phys. Rev. B 48, 7790 (1993)

16 A. Houghton, H. J. Kwon, J. B. Marston, and R. Shankar, 70श0Ф4y03925, 4909 (1994)

"A. Houghton, H. J. Kwon, and J. B. Marston, Adv. Phys. 49, 141 (2000).

18 F. D. M. Haldane, in Proceedings of the International School of Physics "Enrico Fermi," course 121, Varenna, 1992, edited by J. R. Schrieffer and R. Broglia (North-Holland, New York, 1994).

19 D. G. Barci and L. E. Oxman, Phys. Rev. B 67, 205108 (2003)

${ }^{20}$ H. L. Calvo and H. M. Pastawski, Braz. J. of Physics 36, 963 (2006).

${ }^{21}$ C. G. da Silva and B. Koiller, Solid State Communications 40, 215 (1981)

22 J. B. Sokoloff and J. V. José, Phys. Rev. Lett. 49, 334 (1982)

23 E. Domany, S. Alexander, D. Bensimon, and L. P. Kadanoff, Phys. Rev. B 28, 3110 (1983).

24 W. D. Heiss, M. Müller, and I. Rotter, Phys. Rev. E 58, 2894 (1998)

25 I. Rotter,Journal of Physics A: Mathematical and Theoretical 42, 153001 (2009

26 I. Rotter and J. P. Bird, Reports on Progress in Physics 78, 114001 (2015)

27 J. Y. Khoo and I. S. Villadiego, Phys. Rev. B 99, 075434 (2019)

28 J. Nilsson and A. H. Castro Neto, Phys. Rev. B 72, 195104 (2005)

29 D. G. Barci and D. Reyes, Phys. Rev. B 87, 075147 (2013)

30 S. Golkar, D. X. Nguyen, M. M. Roberts, and D. T. Son, Phys. Rev. Lett. 117, 216403 (2016)

31 D. G. Barci, E. Fradkin, and L. Ribeiro, Phys. Rev. B 98, 155146 (2018)

32 R. Shankar, Rev. Mod. Phys. 66, 129 (1994) 

33 W. Metzner, C. Castellani, and C. D.
Castro, https://doi.org/10.1080/000187398243528

34 G. Y. Chitov and D. Sénéchal, Phys. Rev. B 57, 1444 (1998)

35 A. Keleş and E. Zhao, Phys. Rev. A 94, 033616 (2016)

${ }^{36}$ M. Heyl, Reports on Progress in Physics 81, 054001 (2018)

37 N. Nessi, A. Iucci, and M. A. Cazalilla, Phys. Rev. Lett. 113, 210402 (2014)

38 R. M. Fernandes, A. V. Chubukov, and J. Schmalian, Nature Physics 10, 97 EP (2014)

39 J. Li, P. J. Pereira, J. Yuan, Y.-Y. Lv, M.-P. Jiang, D. Lu, Z.-Q. Lin, Y.-J. Liu, J.-F. Wang, L. Li, X. Ke, G. Van Tendeloo, M.-Y. Li, H.-L. Feng, T. Hatano, H.-B. Wang, P.-H. Wu, K. Yamaura,
E. Takayama-Muromachi, J. Vanacken, L. F. Chibotaru, and V. V. Moshchalkov, Nature Communications 8, 1880 (2017)

40 D. G. Barci, E. Fradkin, S. A. Kivelson, and V. Oganesyan, Phys. Rev. B 65, 245319 (2002)

41 C. Wexler and A. T. Dorsey, Phys. Rev. B 64, 115312 (2001).

${ }^{42}$ B. E. Feldman, M. T. Randeria, A. Gyenis, F. Wu, H. Ji, R. J. Cava, A. H. MacDonald, and A. Yazdani, Science 354, 316 (2016), https://science.sciencemag.org/content/354/6310/316.full.pdf

43 C. Chin, R. Grimm, P. Julienne, and E. Tiesinga, Rev. Mod. Phys. 82, 1225 (2010)

44 C. H. Schunck, M. W. Zwierlein, C. A. Stan, S. M. F. Raupach, W. Ketterle, A. Simoni, E. Tiesinga, C. J. Williams, and P. S. Julienne, Phys. Rev. A 71, 045601 (2005). 